\title{
The Turkish experience of poliomyelitis before and after a vaccination campaign
}

\author{
Fitnat Dinçer ${ }^{1}$, Sevim Orkun ${ }^{2}$ and Seyhan Sözay ${ }^{3}$ \\ Physical Medicine and Rehabilitation (PMR), ${ }^{1}$ Hacettepe Univ. School of Medicine Department of PMR, Ankara, \\ Turkey; ${ }^{2}$ Ankara Rehabilitation Center, Ankara, Turkey; ${ }^{3}$ Baskent Univ. Hospital, Ankara, Turkey
}

\begin{abstract}
It is very important that the poliovirus is eradicated as it has caused serious and permanent disabilities during the present century. In order to achieve success in the rehabilitation of poliomyelitis patients the role of vaccination campaigns as an essential part of preventive medical care should be appreciated. After every vaccination campaign successful results have been obtained. Thus we are optimistic that if these vaccination campaigns are carried out successful results will be achieved as was obtained after the 1985 vaccination campaign. As is understood from our report, according to the results of the 1985 vaccination campaign successful long-term results have been achieved. The results of the 1995 campaign will also be seen at a future date as this article does not report them. We appreciate the cooperation and support given by WHO during the staging of these campaigns.
\end{abstract}

Keywords: poliomyelitis; poliovirus vaccine; vaccination campaign; rehabilitation

\section{Introduction}

The polio virus has caused disability in millions of children during this century. Therefore the World Health Organization (WHO) aims to repeat the success that was achieved with chicken pox. WHO is targeting global eradication of poliomyelitis by the year $2000 .{ }^{1}$ Vaccination campaigns and various preventive medicine programs have been carried out for this purpose. The goal is that there should be no clinical case of polio virus infection and no virus in the samples taken from the population and the environment. ${ }^{2}$ In many developing countries, vaccination campaigns and vaccination days are arranged for this purpose.

Outstanding success has been achieved on the American Continent, where no person with poliomyelitis has been reported for 4 years in the southern part of South America. Wild type 3 poliovirus was isolated in the Alberta province of Canada, which was related to the epidemic observed in the Netherlands in 1992 and no paralysis was detected in the investigated group. ${ }^{3}$

In September 1985, a mass vaccination campaign was started in Turkey with the cooperation of The Ministry of Health, WHO, UNICEF and voluntary organizations. Children between the ages of $0-60$ months were vaccinated. Three doses of trivalent oral poliovirus vaccine were given. The same vaccination schedule is still continuing. ${ }^{4}$

Physiatrists have been concerned with the therapy

Correspondence: Prof. F Dinçer M.D. of people with poliomyelitis in former years. Poliomyelitis can cause permanent disability, therefore the treatment of the acute and chronic phases of the disease is essential. Efficacy of the vaccination programs and preventive medicine can be reflected in patient records from rehabilitation departments. Therefore the patient records of the Ankara Rehabilitation Center and physical medicine and rehabilitation (PMR) department of Hacettepe Medical School have been investigated retrospectively.

\section{Materials and methods}

Records of 853 patients admitted to the Ankara Rehabilitation Center and the PMR Department of Hacettepe Medical School between 1980-1990 years were examined. Patients, both outpatient and inpatient, were analyzed according to years. These records were compared with the records of the Ministry of Health, and the efficacy of the vaccination campaign and preventive medicine was evaluated.

\section{Result}

The total number of inpatients and outpatients registered in The Ankara Rehabilitation Center and PMR Department of Hacettepe Medical School for the first time between 1980-1990 (both new and old cases), are shown in Table 1). From 1986, that is after the vaccination campaign, the characteristics of the patients recorded as new, old or unknown in the registers are shown in Table 2. New cases of 
poliomyelitis and the number of deaths from poliomyelitis between 1980-1994 registered by the Ministry of Health are shown in Tables 3 and 4 respectively. The morbidity and mortality rates during the same period are shown in Tables 5 and 6 .

\section{Discussion}

Global eradication of poliomyelitis is targeted by the year 2000. In some regions of the world, eradication of poliomyelitis has been successful, but developing countries cannot approach this target. WHO has categorized countries according to the incidence of poliomyelitis cases and the rate of vaccination. Category A includes countries where none have been recorded for the last 3 years, the poliovirus vaccination rate is over $60 \%$ and a reliable registered system exists. Turkey is listed in Category $\mathrm{C}$ according to the data of

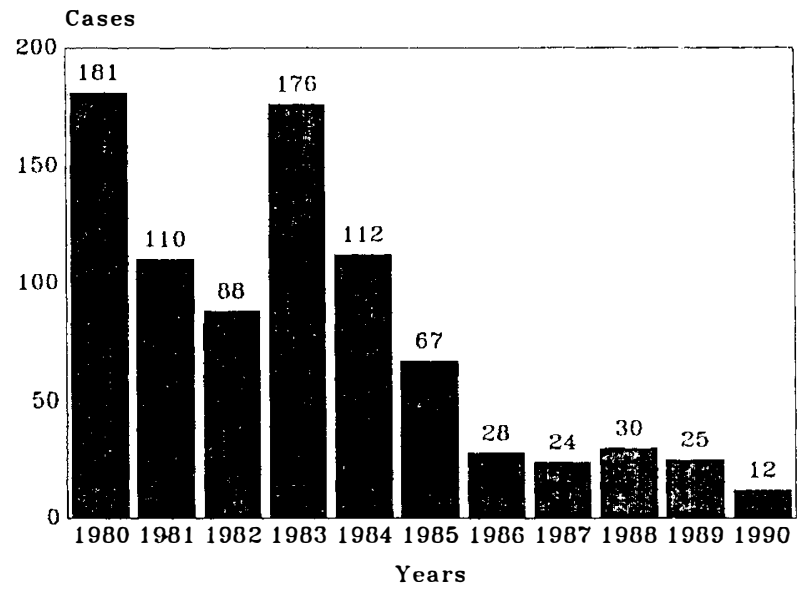

Figure 1 Number of patients presenting annually

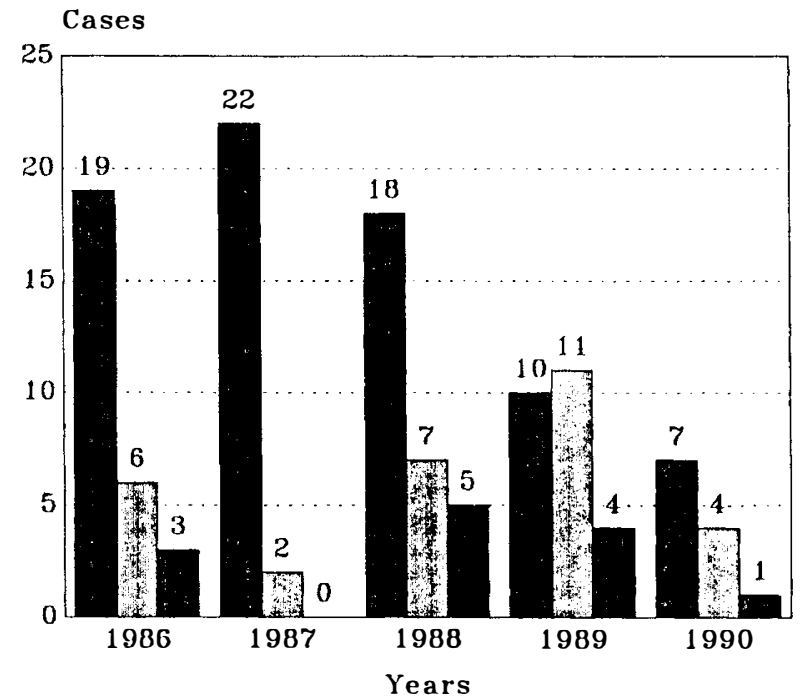

Figure 2 Characteristics of patients after the vaccination campaign $\square$ Old, $\square$ New, $\square$ Unknown

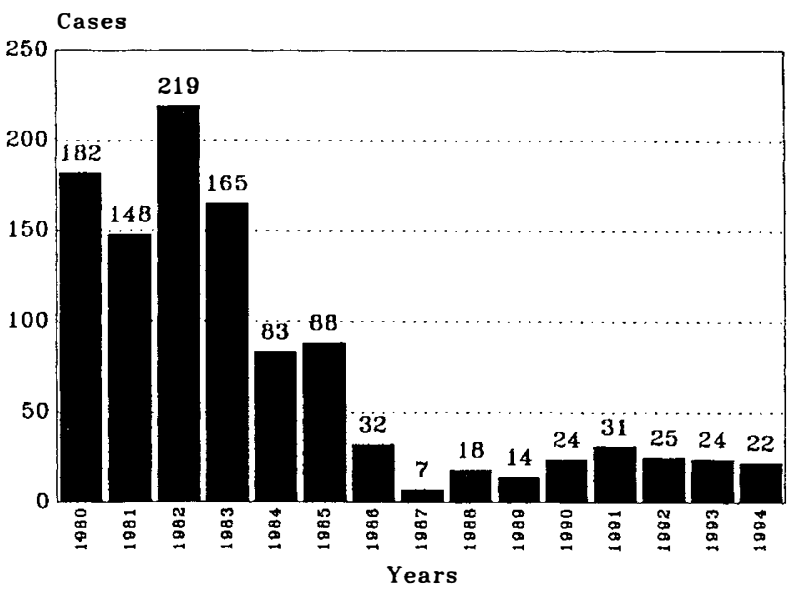

Figure 3 New patients with poliomyelitis discovered between 1980-1994: from Ministry of Health records

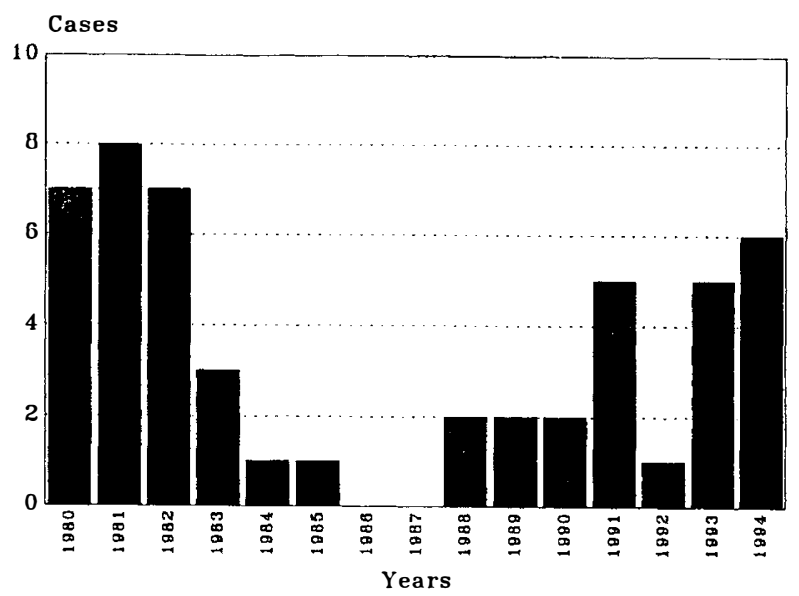

Figure 4 Deaths from poliomyelitis 1980-1994: from Ministry of Health records

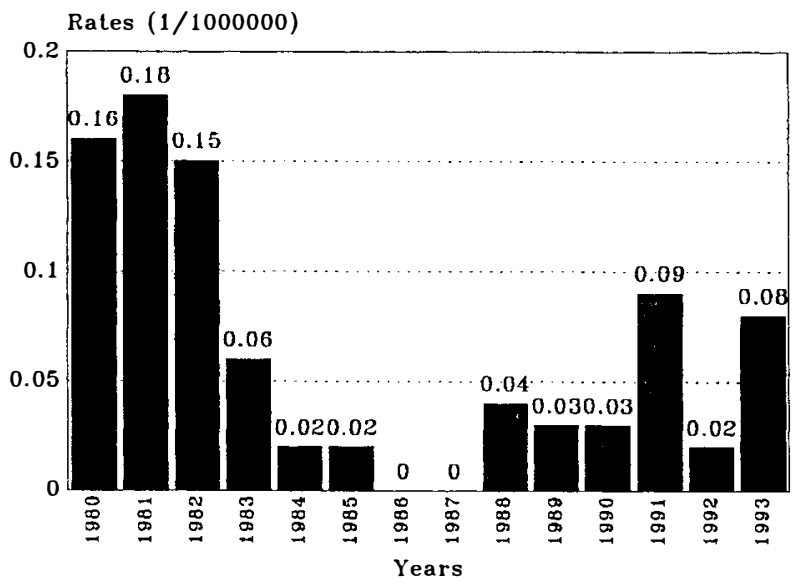

Figure 5 Morbidity rates due to poliomyelitis in 1980-1993: from Ministry of Health records 


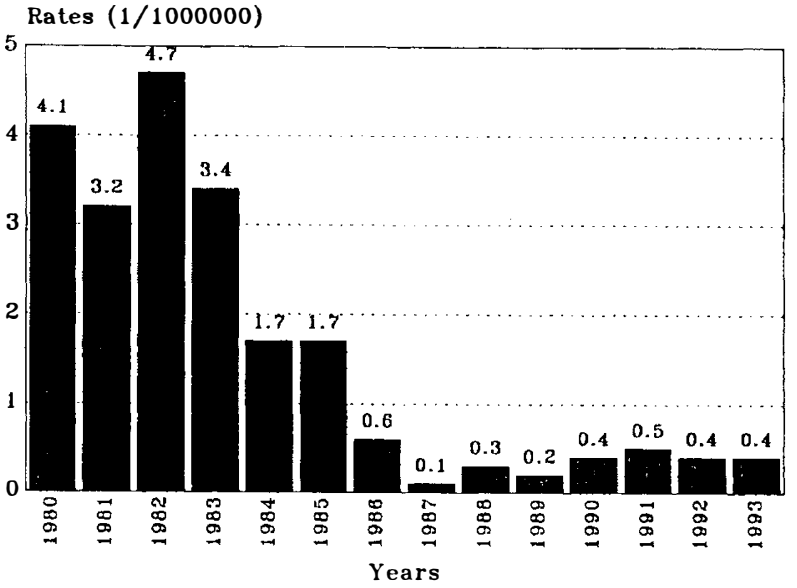

Figure 6 Mortality rates from poliomyelitis in 1980-1993: from Ministry of Health records

1993, with more than 10 patients a year and a poliovirus vaccination rate of about $50 \% .^{5}$ In India, an incidence of acute paralytic poliomyelitis of 20 to $40 / 100,000$ of the population per year, or 200,000 to 400,000 new patients annually, has recently been estimated. ${ }^{6}$ Twenty-three new instances were reported in Turkey in $1994 .^{7}$ The presence of one patient with paralytic poliomyelitis in a region means that the number of polio virus infections without paralysis would be about a hundred to a thousand in the same environment. ${ }^{3}$ The reason for this high incidence might be seasonal and geographical handicaps, or incomplete programming of vaccination, or noncompliance of the cold chain and the epidemic of wild poliomyelitis virus from neighbouring countries especially in the Eastern and Southeastern Anatolian regions. The wild poliomyelitis epidemic in Israel was due to the poor hygenic conditions of the neighbouring countries. Despite the success of the campaign against poliomyelitis with the combined use of oral poliomyelitis vaccine (OPV) and inactivated poliomyelitis vaccine (IPV), infection with the polio virus from neighbouring countries was seen in $1980 .^{8}$

The incidence of poliomyelitis decreased dramatically in the USA with the use of IPV and OPV. Each year fewer than ten patients were reported and these were shown to develop due to vaccine. However in some regions the vaccination campaign was started in order to increase the seroconversion rate, as in developing countries. ${ }^{9}$ A vaccination campaign controlled a poliomyelitis endemic in Gambia, but a few years later a major poliomyelitis epidemic developed. The authors suggest that vaccination campaigns have limited success in controlling an epidemic. Provision of clean water, education in hand washing and building of drainage systems are important together with the application of the vaccine. ${ }^{10}$ Enterovirus infections due to poor environmental health conditions cause compromise polio vaccination. ${ }^{8}$ Poor hygienic condi- tions and a high enterovirus infection rate in the summer hinders the eradication of poliomyelitis in our country.

The enhanced potency of IPV or OPV combined vaccine application decreases the incidence of paralytic poliomyelitis and provides permanent mucosal protection. Two doses of OPV following twice enhanced potency IPV provide systemic and local immunity. ${ }^{11}$ Some suggest that effective seroconversion may be achieved by enhanced potency IPV or OPV only without any change in the vaccination schedule. ${ }^{12}$ The addition of another dose of poliomyelitis vaccine with the rubella vaccine in the twelfth month may help to increase the seroconversion rate and decrease the need for a vaccination campaign or additional vaccination visits.

The vaccination campaign in 1985 in Turkey was successful, as is set out in the tables. However, increases in both new cases and in deaths were still seen, which should not occur if the vaccinations were carried out rigorously. In order to eradicate poliomyelitis in Turkey, vaccination must be carried out regularly, any irregularities must be stopped, the importance of vaccination must be stressed. As is understood from our report according to the results of the 1985 vaccination campaign successful results have been achieved in the long-term. In order to achieve success in the rehabilitation of poliomyelitis patients the role of the vaccination campaigns as an essential part of preventative medical care should be appreciated.

\section{Acknowledgements}

We would like to express our gratitude to Prof Dr Selma Yörükan, Dr Suayip Yalcin and Meral Candan for their valuable assistance.

\section{References}

1 Progress toward global eradication of poliomyelitis, 1988- 1993. $M M W R$ 1994; 43: 499-503.

2 Global poliomyelitis eradication by the year 2000. Manual for the virological investigation of poliomyelitis. EPI and Division of Communicable Diseases, WHO, 1990.

3 Robbins FC. Eradication of polio in the Americans. JAMA 1993; 270 (15): $1857-1859$.

41985 yili hizlandirilmis ve genisletilmis asilama hizmetleri kaydetme ve degerlendirme rehberi, Saḡlik ocaḡi-ACS merkezi hekimleri ilce ve il asilama sorumlulari icin, SSYB.

5 Tumay S, Eminoḡlu N, Demirören M. Polio Eradikasyon Programi. Sürekli Tip Egitimi Dergisi 1994; 3(4): 126-128.

6 Grimby G, Jönsson AT. Disability in poliomyelitis sequelae. Physical Therapy 1994; 74: 415-424.

7 Mass vaccination with oral poliovirus vaccine - Asia and Europe, 1995. JAMA 1995; 273: $1407-1408$.

8 Tulchinsky T, Abed Y, Shaheen S. A ten-year experience in control of poliomyelitis through a combination of live and killed vaccines in two developing areas. Am J Public Health 1989; 79: $1648-1652$.

9 Assessment of undervaccinated children following a mass vaccination campaign-Kansas, 1993. MMWR 1994; 43: 572 573. 
10 Otten MW, Dewing MS, Jaiteh KO. Epidemic poliomyelitis in the Gambia following the control of poliomyelitis as an endemic disease. Am J Epidemiol 1992; 135: 381 - 392.

11 McBean AM, Thomas ML, Albrecht P. Serologic response to oral polio vaccine and enhanced-potency inactivated polio vaccines. Am J Epidemiol 1988; 128: 615-628.
12 Moriniere BJ, van Loom FPL, Rhodes PH. Immunogenicity of a supplemental dose of oral versus inactivated poliovirus vaccine. Lancet 1993; 341: $1545-1550$. 